IMA Journal of Numerical Analysis (2013) 33, 57-79

doi:10.1093/imanum/drs010

Advance Access publication on May 28, 2012

\title{
On conjugate symplecticity of B-series integrators
}

\author{
ERNST HAIRER* AND CHRISTOPHE J. ZBINDEN \\ Section de Mathématiques Université de Genève, 2-4 rue du Lièvre, CH-1211 Genève 4, Switzerland \\ *Corresponding author: ernst.hairer@unige.ch
}

[Received on 15 November 2011; revised on 15 November 2011]

The long-time integration of Hamiltonian differential equations requires special numerical methods. Symplectic integrators are an excellent choice, but there are situations (e.g., multistep schemes or energypreserving methods), where symplecticity is not possible. It is then of interest to study whether the methods are conjugate symplectic and thus have the same long-time behaviour as symplectic methods. This question is addressed in this work for the class of B-series integrators. Algebraic criteria for conjugate symplecticity up to a certain order are presented in terms of the coefficients of the B-series. The effect of simplifying assumptions is investigated. These criteria are then applied to characterize the conjugate symplecticity of implicit Runge-Kutta methods (Lobatto IIIA and Lobatto IIIB) and of energy-preserving collocation methods.

Keywords: conjugate symplecticity; Hamiltonian differential equations; backward error analysis; modified equations; B-series; rooted trees; simplifying assumptions; Lobatto IIIA methods; Lobatto IIIB methods; energy-preserving integrators.

\section{Introduction}

Consider a Hamiltonian differential equation

$$
\dot{y}=J^{-1} \nabla H(y), \quad J=\left(\begin{array}{cc}
0 & I \\
-I & 0
\end{array}\right),
$$

where $J$ is the canonical structure matrix and $H: \mathbb{R}^{2 d} \rightarrow \mathbb{R}$ is sufficiently differentiable ( $d$ is the number of degrees of freedom). The function $H(y)$ is called the Hamiltonian or energy of the system. A classical result by Poincaré tells us that the exact flow, denoted by $\varphi_{t}(y)$, is for every $t$ a symplectic transformation. This means that the derivative with respect to the initial value satisfies

$$
\varphi_{t}^{\prime}(y)^{\mathrm{T}} J \varphi_{t}^{\prime}(y)=J .
$$

For problems with one degree of freedom this property is equivalent to area preservation, and it implies volume preservation of the flow in the general case. Another property of Hamiltonian systems is energy preservation, which means that $H(y(t))$ is constant along solutions of (1.1).

We are interested in the numerical treatment of Hamiltonian systems. In the spirit of geometric numerical integration, the ideal situation would be to have a numerical integrator $y_{n+1}=\Phi_{h}\left(y_{n}\right)$ for which the discrete flow mapping $\Phi_{h}(y)$ is symplectic and which exactly preserves the energy. Unfortunately this is not possible (Ge \& Marsden, 1988; see also Chartier et al., 2006). One is therefore constrained to consider methods satisfying one of these properties and to study how well the other is verified.

An important tool for studying the long-time behaviour of numerical methods is backward error analysis (see Hairer et al., 2006). It tells us that the discrete flow of a numerical integrator $\Phi_{h}(y)$, when 
applied to $\dot{y}=f(y)$, can be (formally) interpreted as the exact flow of a modified differential equation, whose vector field is given as a series in powers of the step size $h$ :

$$
\dot{y}=f(y)+h f_{1}(y)+h^{2} f_{2}(y)+h^{3} f_{3}(y)+\cdots .
$$

If the method is of order $p$, we have $f_{j}(y)=0$ for $1 \leqslant j<p$, so that the perturbation is of size $\mathcal{O}\left(h^{p}\right)$.

For symplectic methods applied to (1.1) the modified differential equation is Hamiltonian,

$$
\dot{y}=J^{-1} \nabla H_{h}(y) \quad \text { with } H_{h}(y)=H(y)+h H_{1}(y)+h^{2} H_{2}(y)+h^{3} H_{3}(y)+\cdots,
$$

with functions $H_{j}(y)$ that are globally defined for B-series integrators. This shows that the discrete flow of symplectic methods has the same qualitative behaviour as the exact flow. Formally, it exactly conserves the modified Hamiltonian $H_{h}(y)$, so that the energy $H(y)$ is nearly conserved with an error bounded by $\mathcal{O}\left(h^{p}\right)$ (without any drift). Moreover, it can be shown (see, e.g., Hairer et al., 2006) that symplectic methods exactly preserve quadratic first integrals of the system and, in the case of nearly integrable systems, they nearly conserve all action variables and have at most a linear error growth in the angle variables.

In the present article we are interested in methods that are not necessarily symplectic but nevertheless have excellent long-time behaviour. We call a numerical method of order $p$ conjugate symplectic up to order $p+r$ (with $r \geqslant 0$ ) if there exists a change of coordinates $z=\chi(y)$ that is $\mathcal{O}\left(h^{p}\right)$ close to the identity such that $\Psi_{h}=\chi \circ \Phi_{h} \circ \chi^{-1}$ satisfies

$$
\Psi_{h}^{\prime}(z)^{\mathrm{T}} J \Psi_{h}^{\prime}(z)=J+\mathcal{O}\left(h^{p+r+1}\right) .
$$

The method $\Psi_{h}$ has the same order as $\Phi_{h}$, and the coefficient functions $f_{j}(z)$ of the corresponding modified differential equation are Hamiltonian for $j<p+r$. Consequently, the error in the energy $H\left(z_{n}\right)$ is bounded by $\mathcal{O}\left(h^{p}\right)+\mathcal{O}\left(t h^{p+r}\right)$, so that no drift can be seen on intervals of length $\mathcal{O}\left(h^{-r}\right)$. The same is true for the near preservation of quadratic first integrals and for the action variables in nearly integrable Hamiltonian systems. Since for a method that is conjugate symplectic up to order $p+r$ we have $y_{n}-z_{n}=\mathcal{O}\left(h^{p}\right)$, the same statements remain true for the numerical approximation $\left\{y_{n}\right\}$.

In Section 2 we start by recalling the definition of B-series, we present the composition law and we give explicit formulas for the $\mathrm{B}$-series representing the modified equation. We also recall algebraic conditions on the coefficients of a B-series that guarantee its symplecticity, and we discuss the B-series that is obtained after conjugation. Section 3 is then devoted to criteria for conjugate symplecticity in terms of the coefficients of the modified differential equation. A recurrence relation counting the number of necessary conditions is given. Analogous criteria in terms of the coefficients of the B-series integrator are then proved in Section 4. For high order the number of order conditions is very high, and they can be handled only with the use of simplifying assumptions. In Section 5 we recall a coordinate-free definition of simplifying assumptions $C(\eta)$ and $D(\zeta)$, and we discuss the simplification of the algebraic criteria for conjugate symplecticity under these simplifying assumptions. Applications of the criteria are the subject of the final Section 6. We discuss the conjugate symplecticity of Lobatto IIIA and Lobatto IIIB Runge-Kutta methods, and we prove that the energy-preserving collocation methods of maximal order $2 s$ are conjugate symplectic up to order $2 s+2$ but not up to a higher order.

\section{B-series theory}

Based on the seminal publication of Butcher (1972), the concept of B-series was introduced in Hairer \& Wanner (1974). It is motivated by the fact that the exact solution of $\dot{y}=f(y)$, as well as the numerical 
solution of nearly all integrators, can be written as a B-series. For a modern treatment of the theory of B-series we refer to Murua (1999), the monograph of Hairer et al. (2006) and the recent article by Chartier et al. (2010). In the following we collect the definitions and the results that will be needed in this work.

Let

$$
T=\{\bullet, \mathcal{\imath}, \mathcal{V}, \mathcal{\jmath}, \mathcal{V}, \mathcal{V}, \mathfrak{\mathcal { V }},\}, \ldots\}
$$

be the set of rooted trees. If it is convenient to consider also the empty tree, we write $T_{0}=T \cup\{\emptyset\}$. We use the notation $\tau=\left[\tau_{1}, \ldots, \tau_{m}\right]$ for the tree that is obtained by grafting the roots of $\tau_{1}, \ldots, \tau_{m} \in T$ to a new vertex which becomes the root of $\tau$. We denote the number of vertices by $|\tau|$ and call it the order of $\tau$. The symmetry coefficient is defined recursively by

$$
\sigma(\bullet)=1, \quad \sigma(\tau)=\sigma\left(\tau_{1}\right) \cdots \sigma\left(\tau_{m}\right) \mu_{1} ! \mu_{2} ! \cdots,
$$

where the integers $\mu_{1}, \mu_{2}, \ldots$ count equal trees among $\tau_{1}, \ldots, \tau_{m}$. For a differential equation $\dot{y}=f(y)$ the corresponding elementary differentials $F(\tau)$ are given by

$$
F(\bullet)(y)=f(y), \quad F(\tau)(y)=f^{(m)}(y)\left(F\left(\tau_{1}\right)(y), \ldots, F\left(\tau_{m}\right)(y)\right) .
$$

For given real coefficients $a(\emptyset)$ and $a(\tau), \tau \in T$, a B-series is a formal series of the form

$$
B(a, y)=a(\emptyset) y+\sum_{\tau \in T} \frac{h^{|\tau|}}{\sigma(\tau)} a(\tau) F(\tau)(y) .
$$

\section{$2.1 \quad$ B-series integrators}

A discrete flow $\Phi_{h}(y)$, whose (formal) Taylor series is of the form (2.2) with $a(\emptyset)=1$, is called a B-series integrator, $\Phi_{h}(y)=B(a, y)$. It is consistent with $\dot{y}=f(y)$ if in addition $a(\bullet)=1$. This is a wide class of numerical methods that comprises all Runge-Kutta methods, the underlying onestep method of multistep methods, the averaged vector field integrator, energy-preserving collocation methods and many more.

The exact time- $h$ flow of $\dot{y}=f(y)$ can be interpreted as a B-series integrator $\varphi_{h}(y)=B(e, y)$ with coefficients

$$
e(\varnothing)=e(\bullet)=1, \quad e(\tau)=\frac{1}{|\tau|} e\left(\tau_{1}\right) \ldots e\left(\tau_{m}\right) \quad \text { for } \tau=\left[\tau_{1}, \ldots, \tau_{m}\right]
$$

A B-series integrator is of order $p$ if its Taylor series matches that of the exact solution up to an error of size $\mathcal{O}\left(h^{p+1}\right)$. Algebraically, this can be expressed as $a(\tau)=e(\tau)$ for all trees with $|\tau| \leqslant p$.

\subsection{Composition law}

Let $B(c, y)$ be a B-series with $c(\varnothing)=1$, so that it is close to the identity mapping. The expressions $F(\tau)(B(c, y))$ can then be expanded into a Taylor series around $y$, and it turns out (see, e.g., Hairer et al., 2006, p. 62) that the composition of B-series satisfies

$$
B(b, B(c, y))=B(c b, y) \quad \text { with }(c b)(\tau)=\sum_{\theta \in \operatorname{OST}(\tau)} b(\theta) c(\tau \backslash \theta)
$$


Here, $\operatorname{OST}(\tau)$ denotes the set of ordered subtrees of $\tau$. The empty tree $\emptyset$ and $\tau$ are in $\operatorname{OST}(\tau)$, as well as trees $\theta$ that are formed by a connected subset of vertices of $\tau$ containing its root. All vertices of $\tau$ are considered different, so that, for example, the tree $\mathcal{f}$ appears twice in the set OST $(\mathcal{V})$. The difference set $\tau \backslash \theta$ consists of those trees that remain when $\theta$ and its adjacent branches are removed from $\tau$, and the expression $c(\tau \backslash \theta)$ is defined as the product $c(\tau \backslash \theta)=\prod_{\delta \in \tau \backslash \theta} c(\delta)$.

The set $G=\left\{a: T_{0} \rightarrow \mathbb{R} ; a(\emptyset)=1\right\}$ provided with the above composition law is called the Butcher group. Let us also mention that (2.4) defines a coproduct which makes the algebra of polynomials with the rooted trees as commuting indeterminates to a Hopf algebra.

\subsection{B-series vector fields and the modified differential equation}

A B-series $B(\alpha, y)$ with coefficients satisfying $\alpha(\emptyset)=0$ is of the form

$$
B(\alpha, y)=h \alpha(\bullet) f(y)+h^{2} \alpha(\boldsymbol{\bullet}) f^{\prime}(y) f(y)+\cdots
$$

and can be interpreted as a vector field. The modified differential equation of a B-series integrator (in the sense of backward error analysis) is such a vector field. To get a relation between the coefficients $a(\tau)$ of the method and the coefficients $\alpha(\tau)$ of the vector field, it is convenient to work with the Lie derivative $\partial_{\alpha} c$ as discussed in Hairer et al. (2006, p. 370). If $y(t)$ is a solution of the differential equation $h \dot{y}(t)=B(\alpha, y(t))$, then we have

$$
h \frac{\mathrm{d}}{\mathrm{d} t} B(c, y(t))=B\left(\partial_{\alpha} c, y(t)\right) \quad \text { with }\left(\partial_{\alpha} c\right)(\tau)=\sum_{\theta \in \operatorname{SP}(\tau)} c(\theta) \alpha(\tau \backslash \theta)
$$

for $|\tau| \geqslant 1$ and $\left(\partial_{\alpha} c\right)(\emptyset)=0$. Here, $\operatorname{SP}(\tau)=\{\theta \in \operatorname{OST}(\tau) ; \tau \backslash \theta$ consists of only one element $\}$ denotes the set of splittings of the tree $\tau$. Higher derivatives can be expressed in terms of iterated applications of the Lie derivative. It then follows from the Taylor series expansion that $h \dot{y}(t)=B(\alpha, y(t))$ is the modified differential equation of the B-series integrator $y_{n+1}=B\left(a, y_{n}\right)$ if and only if

$$
a(\tau)=\sum_{j=1}^{|\tau|} \frac{1}{j !}\left(\partial_{\alpha}^{j-1} \alpha\right)(\tau) .
$$

This formula yields a bijection between the coefficients $a(\tau)$ and $\alpha(\tau)$, which can be used to compute the modified differential equation from the coefficients of the integrator.

\subsection{Criteria for symplecticity}

The symplecticity of a mapping $y \mapsto B(a, y)$ can be characterized in terms of algebraic conditions on the coefficients of the B-series. To this end, we need the Butcher product of two trees $u, v \in T$, which is defined by

$$
u \circ v=\left[u_{1}, \ldots, u_{m}, v\right] \text { for } u=\left[u_{1}, \ldots, u_{m}\right] .
$$

The B-series $B(a, y)$ is symplectic for all Hamiltonian systems if and only if

$$
a(u \circ v)+a(v \circ u)=a(u) a(v) \text { for all } u, v \in T .
$$

The differential equation $h \dot{y}=B(\alpha, y)$ is Hamiltonian whenever $f(y)=J^{-1} \nabla H(y)$ if and only if

$$
\alpha(u \circ v)+\alpha(v \circ u)=0 \text { for all } u, v \in T .
$$


If the coefficients $a(\tau)$ and $\alpha(\tau)$ are related via (2.6), then both conditions (2.7) and (2.8) are equivalent. These statements are discussed in Hairer et al. (2006, Sections VI.7 and XI.9).

\subsection{Conjugation}

We consider a B-series integrator $y_{n+1}=\Phi_{h}\left(y_{n}\right)$ with $\Phi_{h}(y)=B(a, y)$ and a change of coordinates $z=\chi(y)$ that can be written as a B-series,

$$
z=B(c, y) \quad \text { with } c(\emptyset)=1 \text {. }
$$

In the new coordinates the method becomes $z_{n+1}=\Psi_{h}\left(z_{n}\right)$ with $\Psi_{h}=\chi \circ \Phi_{h} \circ \chi^{-1}$. Using the composition law for B-series, this can be expressed as

$$
z_{n+1}=B\left(b, z_{n}\right) \text { with } b=c^{-1} a c .
$$

If $h \dot{y}=B(\alpha, y)$ denotes the modified differential equation corresponding to the method $B(a, y)$, then the modified differential equation for the method $B(b, z)$ is given by

$$
h \dot{z}=B(\beta, z) \quad \text { with } \beta=c^{-1} \partial_{\alpha} c .
$$

This is a consequence of (2.5) because $h \dot{z}=B\left(\partial_{\alpha} c, y\right)=B\left(\partial_{\alpha} c, B\left(c^{-1}, z\right)\right)$.

\section{Conjugate symplecticity in terms of the modified equation}

We consider a B-series integrator $B(a, y)$ of order $p \geqslant 1$. For the coefficients of the corresponding modified differential equation $h \dot{y}=B(\alpha, y)$ this implies that

$$
\alpha(\emptyset)=0, \quad \alpha(\bullet)=1, \quad \alpha(\tau)=0 \quad \text { for } 2 \leqslant|\tau| \leqslant p .
$$

With a view to studying the conjugate symplecticity of numerical integrators in terms of their modified differential equation, we introduce the notation (for $u, v \in T$ )

$$
\alpha(u, v)=\alpha(u \circ v)+\alpha(v \circ u)
$$

The same notation is used for the coefficients $\beta$ of the modified differential equation in the transformed coordinates and for the coefficients $c$ of the transformation.

LEMMA 3.1 In addition to (3.1), assume that the B-series $B(c, y)$ satisfies

$$
c(\emptyset)=1, \quad c(\tau)=0 \quad \text { for } 1 \leqslant|\tau| \leqslant p-1,
$$

so that $B(c, y)=y+\mathcal{O}\left(h^{p}\right)$, and let $\beta$ be given by (2.10). For $u, v \in T$ with $|u|+|v| \leqslant 2 p$ we then have

$$
\alpha(u, v)=\beta(u, v)-\sum_{\hat{v} \in \mathrm{SP}_{*}(v)} c(u, \hat{v})-\sum_{\hat{u} \in \mathrm{SP}_{*}(u)} c(\hat{u}, v),
$$

where $\operatorname{SP}_{*}(\tau)=\{\theta \in \operatorname{SP}(\tau) ;|\theta|=|\tau|-1\}$ is the set of splittings that separate only one tree with one vertex. By convention, $\mathrm{SP}_{*}(\bullet)$ is the empty set, so that the corresponding sums are zero. 
Proof. The assumption on $c$ implies that the conjugated method is also of order $p$, so that the coefficients $\beta$ satisfy the same relations (3.1) as $\alpha$. We now write equation (2.10) as $\partial_{\alpha} c=c \beta$. The assumptions on $\alpha$ and $c$ imply that only the terms with $\theta=\emptyset$ and $|\theta|=|\tau|-1$ give rise to nonvanishing terms in (2.5). Those on $\beta$ and $c$ imply that in the composition law for $c \beta$, only the terms with $\theta=\tau$ and $|\theta|=1$ have to be considered. The relation $\partial_{\alpha} c=c \beta$ thus yields

$$
\alpha(\tau)+\sum_{\theta \in \operatorname{SP}(\tau),|\theta|=|\tau|-1} c(\theta)=\beta(\tau)+\sum_{\theta \in \operatorname{SP}(\tau),|\theta|=1} c(\tau \backslash \theta) \text { for }|\tau| \leqslant 2 p .
$$

For $\tau=u \circ v$ the sum on the right-hand side of (3.4) is empty if $|u| \geqslant 2$, and the set $\{\theta \in \operatorname{SP}(\tau) ;|\theta|=$ $|\tau|-1\}$ is in one-to-one correspondence with $\mathrm{SP}_{*}(u) \cup \mathrm{SP}_{*}(v)$ if $|v| \geqslant 2$. This proves

$$
\alpha(u \circ v)=\beta(u \circ v)-\sum_{\hat{v} \in \mathrm{SP}_{*}(v)} c(u \circ \hat{v})-\sum_{\hat{u} \in \mathrm{SP}_{*}(u)} c(\hat{u} \circ v),
$$

and the statement of the lemma follows for $|u| \geqslant 2$ and $|v| \geqslant 2$.

For $\tau=\bullet \circ v$ the sum on the right-hand side of (3.4) reduces to $c(v)$. For $\tau=u \circ \bullet$ the set $\{\theta \in \operatorname{SP}(\tau) ;|\theta|=|\tau|-1\}$ is in one-to-one correspondence with $\operatorname{SP}_{*}(u) \cup\{u\}$, so that the sum on the left-hand side has an additional term $c(u)$. In the sum $\alpha(\bullet, v)=\alpha(\bullet \circ v)+\alpha(v \circ \bullet)$ these terms cancel and we get (3.3) also in this case.

EXAMPLE 3.2 For $p \geqslant 2$ and $|u|+|v|=3$ we have

$$
\alpha(\bullet, \boldsymbol{\jmath})=\beta(\bullet, \boldsymbol{\ell})-c(\bullet, \bullet)
$$

For $p \geqslant 2$ and $|u|+|v|=4$ we have

$$
\begin{aligned}
& \alpha(\bullet, \mathcal{V})=\beta(\bullet, \boldsymbol{V})-2 c(\bullet, \boldsymbol{\jmath}), \\
& \alpha(\bullet, \boldsymbol{\jmath})=\beta(\bullet, \boldsymbol{\jmath})-c(\bullet, \boldsymbol{\jmath}), \\
& \alpha(\boldsymbol{\jmath}, \boldsymbol{\jmath})=\beta(\boldsymbol{\jmath}, \boldsymbol{\jmath})-2 c(\bullet, \boldsymbol{\jmath})
\end{aligned}
$$

Equations (3.3) can be considered as a linear system for the coefficients $c(u, v)$. For its formulation we let $(T \times T)_{r}=\{(u, v) ;|u|+|v|=r\}$ for $r \geqslant 2$, and we consider the vector space of mappings on $(T \times T)_{r}$,

$$
\mathcal{V}_{r}=\left\{c:(T \times T)_{r} \rightarrow \mathbb{R} ; c(u, v)=c(v, u)\right\}
$$

To compute the dimension of this vector space we consider the formal series

$$
\begin{aligned}
& N(\zeta)=n_{1} \zeta+n_{2} \zeta^{2}+n_{3} \zeta^{3}+\cdots=\zeta(1-\zeta)^{-n_{1}}\left(1-\zeta^{2}\right)^{-n_{2}}\left(1-\zeta^{3}\right)^{-n_{3}} \cdots, \\
& M(\zeta)=m_{2} \zeta^{2}+m_{3} \zeta^{3}+m_{4} \zeta^{4}+\cdots=\frac{1}{2}\left(N(\zeta)^{2}+N\left(\zeta^{2}\right)\right)
\end{aligned}
$$

The coefficient $n_{r}$ denotes the number of trees with $r$ vertices (this formula is due to Cayley and can be found in Hairer et al., 2006, p. 95), and a straightforward computation shows that the coefficient $m_{r}$ is 
TABLE 1 Number of rooted trees of order $r$ and dimension $m_{r}$ of the vector space $\mathcal{V}_{r}$

\begin{tabular}{llllllllllllc}
\hline$r$ & 1 & 2 & 3 & 4 & 5 & 6 & 7 & 8 & 9 & 10 & 11 & 12 \\
\hline$n_{r}$ & 1 & 1 & 2 & 4 & 9 & 20 & 48 & 115 & 286 & 719 & 1842 & 4766 \\
$m_{r}$ & 0 & 1 & 1 & 3 & 6 & 16 & 37 & 96 & 239 & 622 & 1607 & 4235 \\
\hline
\end{tabular}

the dimension of $\mathcal{V}_{r}$. These numbers are given in Table 1 for $r \leqslant 12$. They have also been computed in Celledoni et al. (2010).

We consider the linear mapping $A$ on $\cup_{r \geqslant 2} \mathcal{V}_{r}$, whose restriction $A: \mathcal{V}_{r} \rightarrow \mathcal{V}_{r+1}$ is defined by

$$
(A c)(u, v)=\sum_{\hat{v} \in \mathrm{SP}_{*}(v)} c(u, \hat{v})+\sum_{\hat{u} \in \mathrm{SP}_{*}(u)} c(\hat{u}, v)
$$

so that the condition (3.3) becomes $\alpha(u, v)=\beta(u, v)-(A c)(u, v)$. Since symplecticity of the transformed method is equivalent to $\beta(u, v)=0$ for all $u, v \in T$, the integrator $\Phi_{h}(y)=B(a, y)$ is conjugate symplectic up to order $p+r$ if and only if there exists a B-series $B(c, y)$ satisfying (3.2) such that $\alpha(u, v)=-(A c)(u, v)$ for all pairs of trees with $|u|+|v| \leqslant p+r$. Before we state this as a theorem, we show that the conditions are independent and we prove that the assumption (3.2) can be removed.

LEMma 3.3 The mapping $A: \mathcal{V}_{r} \rightarrow \mathcal{V}_{r+1}$ of (3.5) is injective.

Proof. For a mapping $c:(T \times T)_{r} \rightarrow \mathbb{R}$ we have to prove that the condition $A c=0$ implies $c=0$. It is sufficient to consider pairs of trees satisfying $|u| \leqslant|v|$.

We start with $u=\bullet$, we assume that $(A c)(\bullet, v)=0$ for trees $v$ with $|v|=r$, and we prove by induction on the height of the tree $\hat{v}$ that $c(\bullet, \hat{v})=0$ for trees with $|\hat{v}|=r-1$. We denote by $\mu_{k}$ the unique tree with $k$ vertices and maximal height $k$. The equation $(A c)\left(\bullet, \mu_{r}\right)=c\left(\bullet, \mu_{r-1}\right)$ then proves $c(\bullet, \hat{v})=0$ for $\hat{v}=\mu_{r-1}$. Assume that this relation holds for $\hat{v}$ with height at least $h$. For an arbitrary tree $\hat{v}$ with $r-1$ vertices and height $h-1$ we choose a tree $v$ of height $h$ such that $\hat{v} \in \operatorname{SP}_{*}(v)$. We have $(A c)(\bullet, v)=c(\bullet, \hat{v})$ because further terms in the sum (3.5) vanish by the induction hypothesis. Consequently, $c(\bullet, \hat{v})=0$ for all trees with $|\hat{v}|=r-1$.

We next put $u=\ell$ and apply the same induction argument as above on the height of the tree $\hat{v}$. Then we consider trees of order 3 for $u$, etc.

LEMMA 3.4 Consider a B-series integrator $B(a, y)$ of order $p$ which is conjugate symplectic up to order $p+r$ with $r \geqslant 0$. Then there exists a change of coordinates $z=B(c, y)$ satisfying $B(c, y)=y+\mathcal{O}\left(h^{p}\right)$, such that in the $z$ coordinates the method is symplectic up to order $p+r$.

Proof. Since the method is conjugate symplectic up to order $p+r$, there exists a change of coordinates $z=B(c, y)$ that makes the method symplectic up to order $p+r$. Let $\rho=\min \{|\tau| ; \tau \in T, c(\tau) \neq$ $0\}$. If $\rho \geqslant p$, nothing has to be proved. Therefore, let us assume $\rho<p$. For trees $(u, v)$ satisfying $|u|+|v|=\rho+1$ we have $\alpha(u, v)=0$ (as a consequence of order $p$ ) and $\beta(u, v)=0$ (as a consequence of symplecticity). Lemma 3.1 (with $\rho$ in place of $p$ ) shows that $(A c)(u, v)=0$ for all such pairs of trees, and Lemma 3.3 implies $c\left(u^{*}, v^{*}\right)=0$ for all trees with $\left|u^{*}\right|+\left|v^{*}\right|=\rho$. Consequently, there exists a symplectic mapping $B\left(c_{\rho}, y\right)$ such that $B\left(c_{\rho}, y\right)=B(c, y)+\mathcal{O}\left(h^{\rho+1}\right)$. The transformation $B\left(c c_{\rho}^{-1}, y\right)$ is $\mathcal{O}\left(h^{\rho+1}\right)$ close to the identity and leaves the transformed method symplectic up to order $p+r$. The proof can be repeated until $\rho \geqslant p$ is reached. 
LEMMA 3.5 Consider a symmetric B-series integrator $B(a, y)$ of order $p$ which is conjugate symplectic up to order $p+r$ with $0 \leqslant r \leqslant p$. Then there exists a change of coordinates $z=\chi_{h}(y)=B(c, y)$ satisfying $\chi_{-h}(y)=\chi_{h}(y)$ such that in the $z$ coordinates the method is symplectic up to order $p+r$.

Proof. By Lemma 3.4 it is sufficient to consider transformations $B(c, y)$ that are $\mathcal{O}\left(h^{p}\right)$ close to the identity. For pairs of trees $(u, v)$ with even $|u|+|v|$ we have $\alpha(u, v)=0$ by the symmetry of the method. Lemma 3.1 thus implies $(A c)(u, v)=0$ for all such pairs of trees if $|u|+|v| \leqslant p+r$, and Lemma 3.3 implies $c\left(u^{*}, v^{*}\right)=0$ for all trees with odd $\left|u^{*}\right|+\left|v^{*}\right| \leqslant p+r-1$. The same argument as in the proof of Lemma 3.4 shows that nonzero terms $c(\tau)$ with odd $|\tau| \leqslant p+r-1$ can be removed from the transformation. Nonzero terms $c(\tau)$ with $|\tau| \geqslant p+r$ can also be removed because they do not affect conjugate symplecticity up to order $p+r$.

THEOREM 3.6 A B-series integrator $B(a, y)$ of order $p$ is conjugate symplectic up to order $p+r$ (with $0 \leqslant r \leqslant p)$ if and only if there exist coefficients $c\left(u^{*}, v^{*}\right)$ such that the B-series coefficients of its modified differential equation $h \dot{y}=B(\alpha, y)$ satisfy

$$
\alpha(u, v)=-\sum_{\hat{v} \in \mathrm{SP}_{*}(v)} c(u, \hat{v})-\sum_{\hat{u} \in \mathrm{SP}_{*}(u)} c(\hat{u}, v) \text { for } p<|u|+|v| \leqslant p+r .
$$

Elimination of the coefficients $c\left(u^{*}, v^{*}\right)$ gives exactly $m_{p+r}-m_{p}$ linear relations between the expressions $\alpha(u, v)$. If the integrator is symmetric, the conditions (3.6) are automatically satisfied for trees with even $|u|+|v|$.

Proof. It follows from Lemma 3.4 that (3.2) can be assumed without loss of generality. The equivalence of conjugate symplecticity with (3.6) is then a consequence of Lemma 3.1. Finally, Lemma 3.3 yields the number of additional order conditions, and Lemma 3.5 the statement for symmetric methods.

\section{Conjugate symplecticity in terms of the B-series integrator}

The aim of this section is to translate the criterion of Theorem 3.6 into conditions on the coefficients $a(\tau)$ of the integrator $y_{n+1}=B\left(a, y_{n}\right)$. To this end, we introduce the expression (for $u, v \in T$ )

$$
a(u, v)=a(u \circ v)+a(v \circ u)-a(u) a(v) .
$$

The coefficients $\alpha(\tau)$ of the modified differential equation are related to the coefficients $a(\tau)$ of the method by (2.6). We have to find a similar relation between the expressions $\alpha(u, v)$ for the modified equation and $a(u, v)$ of the B-series, so that the conditions for conjugate symplecticity can be expressed in terms of $a(u, v)$.

The following notation will be convenient: a $j$-fold splitting of a tree $\tau$ is a chain of ordered subtrees

$$
\theta_{0}<\theta_{1}<\cdots<\theta_{j-1}<\theta_{j}=\tau \quad \text { such that } \theta_{l-1} \in \operatorname{SP}\left(\theta_{l}\right), \quad l=1, \ldots, j .
$$

We denote such a $j$-fold splitting by $\Theta=\theta_{0}<\theta_{1}<\cdots<\theta_{j-1}<\theta_{j}$, and we let $\operatorname{SP}^{j}(\tau)$ be the set of all $j$-fold splittings of $\tau$. For a mapping on the set of trees satisfying $\alpha(\emptyset)=0$ we define

$$
\alpha(\Theta)=\alpha\left(\theta_{0}\right) \alpha\left(\theta_{1} \backslash \theta_{0}\right) \cdots \alpha\left(\theta_{j} \backslash \theta_{j-1}\right)
$$

We further consider the set $\operatorname{SP}_{*}^{j}(\tau)=\left\{\Theta \in \operatorname{SP}_{*}^{j}(\tau) ; \theta_{l-1} \in \operatorname{SP}_{*}\left(\theta_{l}\right), l=1, \ldots, j\right\}$ which consists of $j$-fold splittings for which $\left|\theta_{j} \backslash \theta_{j-1}\right|=1$ for all $j$. 
Lemma 4.1 Assume the B-series integrator $B(a, y)$ to be of order $p \geqslant 1$, and let $u$ and $v$ be nonempty trees satisfying $|u|+|v| \leqslant 2 p$. Then we have

$$
a(u, v)=\sum_{j \geqslant 1} \frac{1}{j !} \sum_{\Theta \in \mathrm{SP}_{*}^{j-1}(u \circ v)} \alpha(\hat{u}, \hat{v}),
$$

where $\hat{u}$ and $\hat{v}$ are nonempty ordered subtrees of $u$ and $v$, respectively, such that the smallest tree in the splitting $\Theta$ is $\theta_{0}=\hat{u} \circ \hat{v}$. Splittings, for which $\theta_{0}$ is not of this form, are not considered in (4.1).

Proof. Iteratively applying formula (2.5) for the Lie derivative $\partial_{\alpha}$ we obtain

$$
\left(\partial_{\alpha}^{j-1} \alpha\right)(\tau)=\sum_{\Theta \in \mathrm{SP}^{j-1}(\tau)} \alpha(\Theta) .
$$

For a pair of trees $(u, v)$ we split this sum into

$$
\left(\partial_{\alpha}^{j-1} \alpha\right)(u \circ v)=\sum_{\Theta \in \mathrm{SP}_{+}^{j-1}(u \circ v)} \alpha(\Theta)+\sum_{\Theta \in \mathrm{SP}_{-}^{j-1}(u \circ v)} \alpha(\Theta),
$$

where $\mathrm{SP}_{+}^{j-1}(u \circ v)$ denotes the set of $(j-1)$-fold splittings for which the root of $v$ belongs to $\theta_{0}$, and $\mathrm{SP}_{-}^{j-1}(u \circ v)$ is the set of those splittings for which the root of $v$ does not belong to $\theta_{0}$, i.e., there is a separation between the roots of $u$ and $v$ for splittings in $\mathrm{SP}_{-}^{j-1}(u \circ v)$. We write the second sum as

$$
\sum_{\Theta \in \mathrm{SP}_{-}^{j-1}(u \circ v)} \alpha(\Theta)=\sum_{l=1}^{j-1}\left(\begin{array}{l}
j-1 \\
l-1
\end{array}\right) \sum_{\Theta_{u} \in \mathrm{SP}^{l-1}(u)} \alpha\left(\Theta_{u}\right) \sum_{\Theta_{v} \in \mathrm{SP}^{j-l-1}(v)} \alpha\left(\Theta_{v}\right)
$$

Here, $l-1$ denotes the number of branches removed from $u$ and $j-l-1$ the number of branches removed from $v$. The binomial coefficient counts all possible $(j-1)$-fold splittings of $u \circ v$ that reduce to fixed splittings $\Theta_{u}$ and $\Theta_{v}$ for $u$ and $v$, respectively. Similarly, we have

$$
\sum_{\Theta \in \mathrm{SP}_{-}^{j-1}(v \circ u)} \alpha(\Theta)=\sum_{l=1}^{j-1}\left(\begin{array}{c}
j-1 \\
j-l-1
\end{array}\right) \sum_{\Theta_{u} \in \mathrm{SP}^{l-1}(u)} \alpha\left(\Theta_{u}\right) \sum_{\Theta_{v} \in \mathrm{SP}^{j-l-1}(v)} \alpha\left(\Theta_{v}\right) .
$$

As a consequence of the binomial identity $\left(\begin{array}{l}j-1 \\ l-1\end{array}\right)+\left(\begin{array}{c}j-1 \\ l\end{array}\right)=\left(\begin{array}{l}j \\ l\end{array}\right)$ we thus obtain

$$
\sum_{\Theta \in \mathrm{SP}_{-}^{j-1}(u \circ v)} \alpha(\Theta)+\sum_{\Theta \in \mathrm{SP}_{-}^{j-1}(v \circ u)} \alpha(\Theta)=\sum_{l=1}^{j-1}\left(\begin{array}{l}
j \\
l
\end{array}\right) \sum_{\Theta_{u} \in \mathrm{SP}^{l-1}(u)} \alpha\left(\Theta_{u}\right) \sum_{\Theta_{v} \in \mathrm{SP}^{j-l-1}(v)} \alpha\left(\Theta_{v}\right) .
$$

On the other hand, we have

$$
a(u)=\sum_{j \geqslant 1} \frac{1}{j !}\left(\partial_{\alpha}^{j-1} \alpha\right)(u)=\sum_{j \geqslant 1} \frac{1}{j !} \sum_{\Theta \in \mathrm{SP}^{j-1}(u)} \alpha(\Theta)
$$


and a similar formula for $a(v)$. Using the Cauchy product of two series, this implies that

$$
\sum_{j \geqslant 2} \frac{1}{j !}\left(\sum_{\Theta \in \mathrm{SP}_{-}^{j-1}(u \circ v)} \alpha(\Theta)+\sum_{\Theta \in \mathrm{SP}_{-}^{j-1}(v \circ u)} \alpha(\Theta)\right)=a(u) a(v) .
$$

Note that $\mathrm{SP}_{-}^{j-1}(u \circ v)$ is empty for $j=1$. It therefore follows from (4.2) that

$$
a(u, v)=\sum_{j \geqslant 1} \frac{1}{j !}\left(\sum_{\Theta \in \mathrm{SP}_{+}^{j-1}(u \circ v)} \alpha(\Theta)+\sum_{\Theta \in \mathrm{SP}_{+}^{j-1}(v \circ u)} \alpha(\Theta)\right) .
$$

If the method is of order $p$ and if $|u|+|v| \leqslant 2 p$, a splitting $\Theta$ gives a nonzero contribution only if all factors in the product $\alpha(\Theta)$ are 1 , with the exception of $\alpha\left(\theta_{0}\right)$. This means that $\Theta \in \mathrm{SP}_{*}^{j-1}$. Writing $\theta_{0}=\hat{u} \circ \hat{v}$ concludes the proof.

Combining the statements of Theorem 3.6 and Lemma 4.1 yields the desired criterion for conjugate symplecticity.

THEOREM 4.2 A B-series integrator $B(a, y)$ of order $p$ is conjugate symplectic up to order $p+r$ (with $0 \leqslant r \leqslant p)$ if and only if there exist coefficients $c(\hat{u}, \hat{v})$ such that

$$
a(u, v)=-\sum_{j \geqslant 1} \frac{1}{j !} \sum_{\Theta \in \operatorname{SP}_{*}^{j}(u \circ v)} c(\hat{u}, \hat{v}) \quad \text { for } p<|u|+|v| \leqslant p+r .
$$

Here, $\hat{u}$ and $\hat{v}$ are nonempty ordered subtrees of $u$ and $v$, respectively, such that the smallest tree in the splitting $\Theta$ is $\theta_{0}=\hat{u} \circ \hat{v}$. Splittings for which $\theta_{0}$ is not of this form are not considered in (4.4).

If the integrator is symmetric, conditions (4.4) are automatically satisfied for trees with even $|u|+$ $|v|$.

The following particular cases (of low order) were first obtained in the thesis of Leone (2000); see also Hairer et al. (2006, Section VI.8.1). We eliminate the parameters $c\left(u^{*}, v^{*}\right)$ to get conditions on the B-series coefficients only.

EXAMPLE 4.3 Every B-series method of order 2 is conjugate symplectic up to order 3. It is conjugate symplectic up to order 4 if and only if

$$
a(\bullet, \mathcal{V})-2 a(\bullet, \boldsymbol{\jmath})=0, \quad a(\boldsymbol{\jmath}, \boldsymbol{\jmath})-2 a(\bullet, \boldsymbol{\jmath})=0
$$

EXAMPLE 4.4 For a method of order $p$ there are $m_{p+1}-m_{p}$ (with $m_{r}$ taken from Table 1) additional order conditions for being conjugate symplectic up to order $p+1$ (up to $p+2$ for symmetric methods). For $p=4$ we have the three conditions

$$
\begin{aligned}
& 2 a(\bullet, \mathfrak{V})-6 a(\bullet, \mathfrak{V})+3 a(\bullet, \mathfrak{\bigvee})=0, \quad a(\bullet, \mathfrak{\bigvee})-2 a(\bullet, \mathfrak{\}})=0 \\
& a(\bullet, \mathfrak{V})-3 a(\bullet, \mathfrak{\ell})-3 a(\boldsymbol{\jmath}, \mathcal{V})+6 a(\boldsymbol{\jmath}, \boldsymbol{\jmath})=0 \text {. }
\end{aligned}
$$


For a method of order 6 this results in 21 additional conditions, and this number increases rapidly for higher orders. The practical investigation of conjugate symplecticity up to a high order is therefore only possible with the use of simplifying assumptions.

\section{Simplifying assumptions}

Simplifying assumptions play an important role in the construction of high-order Runge-Kutta methods. They reduce the number of order conditions. In this section we give a coordinate-free definition (i.e., independent of the Runge-Kutta coefficients) of the simplifying assumptions $C(\eta)$ and $D(\zeta)$, which is originally due to Butcher (1972); see also Butcher \& Chan (2002). We then study their effect on the conditions for conjugate symplecticity.

Besides the Butcher product $u \circ v$ of two trees, we consider the commutative merging product which, for $u=\left[u_{1}, \ldots, u_{m}\right]$ and $v=\left[v_{1}, \ldots, v_{l}\right]$, is defined by

$$
u \times v=\left[u_{1}, \ldots, u_{m}, v_{1}, \ldots, v_{l}\right]
$$

Furthermore, we introduce the notation $\tau_{k}$ for the bushy tree with $k$ vertices (the unique tree of height 1). We have, for example, $\tau_{1}=\bullet, \tau_{2}=\boldsymbol{\ell}, \tau_{3}=\mathcal{V}, \ldots$

\subsection{Simplifying assumption $C(\eta)$}

DEFinition 5.1 (Partial order on $T$ ) We denote by $\leqslant_{\eta}$ the smallest order relation satisfying

$$
\left[u_{1}^{*}, \ldots, u_{m}^{*}\right] \times \tau_{k+1} \leqslant \eta\left[u_{1}, \ldots, u_{m}\right] \circ \tau_{k},
$$

where $u_{j}^{*} \leqslant \eta_{j}$ for $j=1, \ldots, m$ (with $m \geqslant 0$ ) and $0 \leqslant k \leqslant \eta$ (see Fig. 1).

We note that $m=0$ yields $\tau_{k+1} \leqslant \eta_{\eta}\left[\tau_{k}\right]$ for $k \leqslant \eta$, which is the starting point for the construction of pairs satisfying $u^{*} \leqslant_{\eta} u$. For $k=0$ the relation simply reads $\left[u_{1}^{*}, \ldots, u_{m}^{*}\right] \leqslant_{\eta}\left[u_{1}, \ldots, u_{m}\right]$. By definition, the relation $\leqslant_{\eta}$ is reflexive, transitive and antisymmetric. It is not a total order, and it only compares trees having the same number of vertices.

Definition 5.2 A B-series $B(a, y)$ is said to satisfy the simplifying assumption $C(\eta)$ if

$$
\frac{a\left(u^{*}\right)}{e\left(u^{*}\right)}=\frac{a(u)}{e(u)} \quad \text { whenever } u^{*} \leqslant \eta u .
$$

Here, the $e(u)$, given in (2.3), are the B-series coefficients of the exact flow.
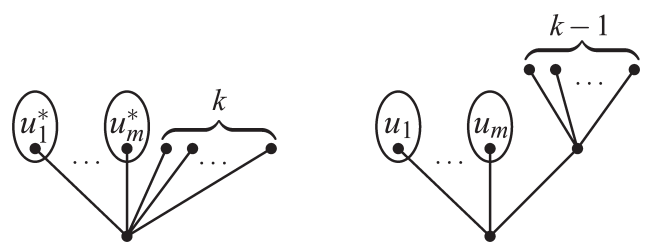

FIG. 1. Trees involved in the simplifying assumption $C(\eta)$. 
LEMMA 5.3 If a B-series $B(a, y)$ satisfies the simplifying assumption $C(\eta)$, then we have

$$
\frac{a\left(u^{*}, v^{*}\right)}{e\left(u^{*}\right) e\left(v^{*}\right)}=\frac{a(u, v)}{e(u) e(v)} \quad \text { whenever } u^{*} \leqslant_{\eta} u, v^{*} \leqslant \eta v .
$$

If the method $B(a, y)$ is of order $p$ and satisfies $C(\eta)$, then the coefficients of its modified differential equation $h \dot{y}=B(\alpha, y)$ satisfy, for $|u|+|v| \leqslant 2 p$,

$$
\frac{\alpha\left(u^{*}, v^{*}\right)}{e\left(u^{*}\right) e\left(v^{*}\right)}=\frac{\alpha(u, v)}{e(u) e(v)} \quad \text { whenever } u^{*} \leqslant_{\eta} u, v^{*} \leqslant_{\eta} v .
$$

Proof. Note that $u^{*} \leqslant_{\eta} u$ and $v^{*} \leqslant_{\eta} v$ imply $\left|u^{*}\right|=|u|,\left|v^{*}\right|=|v|$ and $u^{*} \circ v^{*} \leqslant_{\eta} u \circ v$. The first statement thus follows from the relation $(|u|+|v|) e(u \circ v)=|u| e(u) e(v)$.

The proof of the second statement is by induction on $\rho=|u|+|v|$ and invoking the formula of Lemma 4.1. For $\rho \leqslant p$ the statement is trivial because $\alpha\left(u^{*}, v^{*}\right)=\alpha(u, v)=0$. For $\rho=p+1$ it follows from (5.1) because in this case $\alpha(u, v)=a(u, v)$ and $\alpha\left(u^{*}, v^{*}\right)=a\left(u^{*}, v^{*}\right)$. We now assume that (5.2) holds for pairs $(\hat{u}, \hat{v})$ satisfying $|\hat{u}|+|\hat{v}| \leqslant \rho$, and we consider $u^{*} \leqslant \eta u, v^{*} \leqslant{ }_{\eta} v$ satisfying $|u|+|v|=\rho+1 \leqslant 2 p$. Without loss of generality, we assume $u^{*}=u$ and that $v^{*}$ differs from $v$ only by the fact that one branch $\left[\tau_{k}\right]$ (with $\left.k \leqslant \eta\right)$ in $v$ is replaced by $\tau_{k+1}$ in $v^{*}$.

Lemma 4.1 yields

$$
\frac{a(u, v)}{e(u) e(v)}=\frac{\alpha(u, v)}{e(u) e(v)}+\sum_{j \geqslant 2} \frac{1}{j !} \sum_{\Theta \in \mathrm{SP}_{*}^{j-1}(u \circ v)} \frac{\alpha(\hat{u}, \hat{v})}{e(u) e(v)}
$$

and a similar formula for $\left(u^{*}, v^{*}\right)$. We consider those $\Theta \in \operatorname{SP}_{*}^{j-1}(u \circ v)$, for which $l$ branches are removed from the highlighted subtree $\left[\tau_{k}\right]$ and the other splittings are fixed. There are $(k-1) \cdots(k-l)$ possible $(j-1)$-fold splittings of this kind if $l<k,(k-1) \cdots 2 \cdot 1$ if $l=k$ and 1 if $l=0$. Similarly, we consider splittings $\Theta^{*} \in \mathrm{SP}_{*}^{j-1}\left(u^{*} \circ v^{*}\right)$ for which $l$ branches are removed from the highlighted subtree $\tau_{k+1}$ and the other splittings are exactly the same as above. There are $k \cdots(k-l+1)$ possible $(j-1)$-fold splittings if $l>0$ and 1 if $l=0$. As in Lemma 4.1, we denote the smallest trees of the splittings by $\hat{u} \circ \hat{v}$ and $\hat{u}^{*} \circ \hat{v}^{*}$, respectively. The sum in (5.3) over the considered splittings is, for $l<k$,

$$
(k-1) \cdots(k-l) \frac{\alpha(\hat{u}, \hat{v})}{e(u) e(v)}=(k-1) \cdots(k-l) \frac{e(\hat{u}) e(\hat{v})}{e(u) e(v)} \frac{\alpha(\hat{u}, \hat{v})}{e(\hat{u}) e(\hat{v})}
$$

for the pair $(u, v)$, and for the pair $\left(u^{*}, v^{*}\right)$ it is

$$
k \cdots(k-l+1) \frac{\alpha\left(\hat{u}^{*}, \hat{v}^{*}\right)}{e\left(u^{*}\right) e\left(v^{*}\right)}=k \cdots(k-l+1) \frac{e\left(\hat{u}^{*}\right) e\left(\hat{v}^{*}\right)}{e\left(u^{*}\right) e\left(v^{*}\right)} \frac{\alpha\left(\hat{u}^{*}, \hat{v}^{*}\right)}{e\left(\hat{u}^{*}\right) e\left(\hat{v}^{*}\right)} .
$$

Since $u^{*}=u, \hat{u}^{*}=\hat{u}$ and

$$
e(v)=\frac{1}{k} e\left(v^{*}\right), \quad e(\hat{v})=\frac{1}{k-l} e\left(\hat{v}^{*}\right)
$$

(the second relation is replaced by $e(\hat{v})=e\left(\hat{v}^{*}\right)$ if $l=k$ ), it follows from the induction hypothesis that both expressions (5.4) and (5.5) are the same. This implies that the double sum in (5.3) is the same for $(u, v)$ and for $\left(u^{*}, v^{*}\right)$. Consequently, (5.1) implies (5.2). 
Lemma 5.4 Consider a B-series integrator $B(a, y)$ of order $p$ satisfying $C(\eta)$ and assume that the change of coordinates $z=B(c, y)$ transforms it into a method that is symplectic up to order $p+r$ (with $0 \leqslant r \leqslant p)$. Then the coefficients of the transformation $B(c, y)$ satisfy

$$
\frac{c\left(u^{*}, v^{*}\right)}{e\left(u^{*}\right) e\left(v^{*}\right)}=\frac{c(u, v)}{e(u) e(v)} \quad \text { whenever } u^{*} \leqslant_{\eta} u, v^{*} \leqslant \eta v \text { and }|u|+|v|<p+r .
$$

Proof. Due to the symmetry of the coefficients $c(u, v)$ we can assume without loss of generality that $u^{*}=u$. In the following we combine the ideas of the proofs of Lemmas 3.3 and 5.3.

We put $u=\bullet$, and we consider trees of the form $v=w \circ \tau_{k}$ and $v^{*}=w \times \tau_{k+1}$ with $2 \leqslant k \leqslant \eta$ and $|w|+k<p+r$. For $w=\mu_{s}$ (the unique tree with $s$ vertices and of height $s$ ) it follows from Theorem 3.6 that

$$
\begin{aligned}
\alpha\left(\bullet, \mu_{s} \circ \tau_{k}\right) & =-c\left(\bullet, \mu_{s-1} \circ \tau_{k}\right)-(k-1) c\left(\bullet, \mu_{s} \circ \tau_{k-1}\right), \\
\alpha\left(\bullet, \mu_{s} \times \tau_{k+1}\right) & =-c\left(\bullet, \mu_{s-1} \times \tau_{k+1}\right)-k c\left(\bullet, \mu_{s} \times \tau_{k}\right) .
\end{aligned}
$$

For $k=2$ we have $\mu_{s} \circ \tau_{1}=\mu_{s} \times \tau_{2}$, so that $\alpha\left(\bullet, \mu_{s} \circ \tau_{2}\right)=e\left(\tau_{2}\right) \alpha\left(\bullet, \mu_{s} \times \tau_{3}\right)$ (which follows from Lemma 5.3) implies $c\left(\bullet, \mu_{s-1} \circ \tau_{2}\right)=e\left(\tau_{2}\right) c\left(\bullet, \mu_{s-1} \times \tau_{3}\right)$ for all $s$. Using this result we can treat the case $k=3$ similarly. By an induction argument we then obtain $c\left(\bullet, \mu_{s-1} \circ \tau_{k}\right)=e\left(\tau_{k}\right) c\left(\bullet, \mu_{s-1} \times \tau_{k+1}\right)$ for $2 \leqslant k \leqslant \eta$. We next let $w$ be a tree with $s$ vertices and height $s-1$, and we choose a tree $\tilde{w}$ of height $s$ such that $w \in \operatorname{SP}_{*}(\tilde{w})$. We apply Theorem 3.6 to the pairs of trees $\left(\bullet, \tilde{w} \circ \tau_{k}\right)$ and $\left(\bullet, \tilde{w} \times \tau_{k+1}\right)$, and the same induction argument over $k$ yields the statement of the lemma. As in the proof of Lemma 3.3 we decrease the height of the trees one by one, so that (5.6) is proved for the case $u=\bullet, v=w \circ \tau_{k}$ and $v^{*}=w \times \tau_{k+1}$ with $2 \leqslant k \leqslant \eta$ and $|w|+k<p+r$.

We still keep $u=\bullet$, but we consider trees $v=x \circ\left(w \circ \tau_{k}\right)$ and $v^{*}=x \circ\left(w \times \tau_{k+1}\right)$ with $2 \leqslant k \leqslant \eta$ and $|x|+|w|+k<p+r$. In this situation the proof proceeds by induction on the number of vertices of $x$. For $x=\bullet$ the proof is precisely the same as above. For an arbitrary tree $x$ the formula of Theorem 3.6 yields additional summands, where $x$ is replaced by a subtree belonging to $\operatorname{SP}_{*}(x)$ which can be treated by the induction hypothesis. Up to this point, we have shown the statement of the lemma for $u=\bullet$ and for arbitrary $v$ and $v^{*}$.

We next put $u=$. Here, the application of Theorem 3.6 yields terms where the first argument of $c$ is - (they have already been treated and the statement of the lemma can be used) and terms where the first argument is $\int$. For these terms, the above multiple induction argument has to be repeated. The same happens for trees $u$ with more than two vertices. This then completes the proof of the lemma.

REMARK 5.5 The proof of the previous lemma shows that under the simplifying assumption $C(\eta)$ the condition (3.6) of Theorem 3.6 (or equivalently condition (4.4) of Theorem 4.2) for a pair of trees $(u, v)$ is identical to that for $\left(u^{*}, v^{*}\right)$ if $u^{*} \leqslant{ }_{\eta} u$ and $v^{*} \leqslant{ }_{\eta} v$. This considerably reduces the number of additional order conditions and makes it possible to treat methods of high order.

Lemma 5.4 also shows that not only the number of order conditions for conjugate symplecticity are reduced but also the number of free parameters in the transformation $B(c, y)$.

\subsection{Simplifying assumption $D(\zeta)$}

Definition 5.6 A B-series $B(a, y)$ is said to satisfy the simplifying assumption $D(\zeta)$ if

$$
a\left(\tau_{k} \circ v\right)=e\left(\tau_{k}\right)\left(a(v)-a\left(\tau_{k+1} \times v\right)\right) \quad \text { for } k \leqslant \zeta \text { and all } v \in T \text {, see Fig. } 2
$$



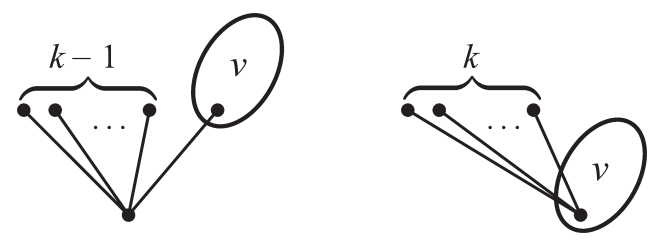

FIG. 2. Trees involved in the simplifying assumption $D(\zeta)$.

The following result shows the simplification by $D(\zeta)$ of the order conditions for conjugate symplecticity. It is an extension of Hairer (2011, Theorem 3).

LEMMA 5.7 If a B-series integrator $B(a, y)$ satisfies the simplifying assumptions $D(\zeta)$ and $C(\eta)$, then we have

$$
a(u, v)=0 \quad \text { for }|u| \leqslant \min (\eta, \zeta) \text { and all } v \in T .
$$

Proof. The simplifying assumption $C(\eta)$ implies $a\left(v \circ \tau_{k}\right)=e\left(\tau_{k}\right) a\left(v \times \tau_{k+1}\right)$ for $k \leqslant \eta$. Together with the simplifying assumption $D(\zeta)$, this yields $a\left(\tau_{k}, v\right)=0$ for $k \leqslant \min (\eta, \zeta)$. The general statement then follows from Lemma 5.3 because the simplifying assumption $C(\eta)$ implies $\tau_{k} \leqslant \eta u$ for all trees $u$ with $|u|=k \leqslant \eta$.

LEMMA 5.8 Consider a B-series integrator $B(a, y)$ of order $p$ satisfying $C(\eta)$ and $D(\zeta)$, and assume that the change of coordinates $z=B(c, y)$ transforms it into a method that is symplectic up to order $p+r$ (with $0 \leqslant r \leqslant p$ ). Then the coefficients of the transformation $B(c, y)$ satisfy

$$
c(u, v)=0 \quad \text { for }|u| \leqslant \min (\eta, \zeta) \text { and }|u|+|v|<p+r .
$$

Proof. The proof is the same as that for Lemma 3.3. We have to stop when the bound $\min (\eta, \zeta)$ is reached for $|u|$.

\subsection{Conjugate symplecticity under simplifying assumptions}

In view of our application in Section 6 we discuss the reduction and simplification of the order conditions for conjugate symplecticity for a few typical situations.

THEOREM 5.9 Consider a symmetric B-series integrator of order $p=2 s-2(s \geqslant 2)$ that satisfies the simplifying assumptions $C(s)$ and $D(s-2)$.

- It is always conjugate symplectic up to order $2 s$.

- For $s \geqslant 3$ it is conjugate symplectic up to order $2 s+2$ if and only if

$$
a\left(\tau_{s-1}, \tau_{s+2}\right)=(s+1) a\left(\tau_{s-1},\left[\tau_{s+1}\right]\right) .
$$

Proof. The condition $a\left(\tau_{s-1}, \tau_{s}\right)=-(s-1) c\left(\tau_{s-1}, \tau_{s-1}\right)$ is the only one that has to be satisfied for conjugate symplecticity up to order $2 s-1$. It can always be satisfied with a suitable choice of $B(c, y)$. Due to the symmetry of the method, it is automatically conjugate symplectic up to order $2 s$. 
For conjugate symplecticity up to order $2 s+2$ the simplifying assumptions imply that we only have to consider the conditions

$$
\begin{aligned}
a\left(\tau_{s-1}, \tau_{s+2}\right) & =-(s+1) c\left(\tau_{s-1}, \tau_{s+1}\right)-\left(\begin{array}{c}
s+1 \\
3
\end{array}\right) c\left(\tau_{s-1}, \tau_{s-1}\right), \\
a\left(\tau_{s-1},\left[\tau_{s+1}\right]\right) & =-s c\left(\tau_{s-1},\left[\tau_{s}\right]\right)-\left(\begin{array}{l}
s \\
3
\end{array}\right) c\left(\tau_{s-1},\left[\tau_{s-2}\right]\right), \\
a\left(\tau_{s}, \tau_{s+1}\right) & =-(s-1) c\left(\tau_{s-1}, \tau_{s+1}\right)-s c\left(\tau_{s}, \tau_{s}\right)-(s-1)\left(\begin{array}{l}
s \\
2
\end{array}\right) c\left(\tau_{s-1}, \tau_{s-1}\right) .
\end{aligned}
$$

The last equation can be satisfied by fixing $c\left(\tau_{s}, \tau_{s}\right)$. By Lemma 5.4 , we have $c\left(\tau_{s-1},\left[\tau_{s}\right]\right)=$ $\frac{1}{s} c\left(\tau_{s-1}, \tau_{s+1}\right)$ and $c\left(\tau_{s-1},\left[\tau_{s-2}\right]\right)=\frac{1}{s-2} c\left(\tau_{s-1}, \tau_{s-1}\right)$ so that the first two relations give the condition (5.9).

Note that the conditions of Example 4.4 are automatically satisfied, if the simplifying assumption $C(3)$ is satisfied.

THEOREM 5.10 Consider a symmetric B-series integrator of order $p=2 s-2(s \geqslant 2)$ that satisfies the simplifying assumptions $C(s-2)$ and $D(s)$.

- It is conjugate symplectic up to order $2 s$ if and only if

$$
a\left(\tau_{s-1}, \tau_{s}\right)=(s-1) a\left(\tau_{s-1},\left[\tau_{s-1}\right]\right) .
$$

Proof. The conditions for conjugate symplecticity up to order $2 s$ are

$$
\begin{gathered}
a\left(\tau_{s-1}, \tau_{s}\right)=-(s-1) c\left(\tau_{s-1}, \tau_{s-1}\right), \\
a\left(\tau_{s-1},\left[\tau_{s-1}\right]\right)=-(s-2) c\left(\tau_{s-1},\left[\tau_{s-2}\right]\right) .
\end{gathered}
$$

Since $c\left(\tau_{s-1},\left[\tau_{s-2}\right]\right)=\frac{1}{s-2} c\left(\tau_{s-1}, \tau_{s-1}\right)$, this proves the statement.

The first statement of the next theorem is one of the main results in Hairer (2011). The criterion for conjugate symplecticity up to order $2 s+4$ is new.

THEOREM 5.11 Consider a symmetric B-series integrator of order $p=2 s(s \geqslant 1)$ that satisfies the simplifying assumptions $C(s)$ and $D(s-1)$.

- It is always conjugate symplectic up to order $2 s+2$.

- For $s \geqslant 2$ it is conjugate symplectic up to order $2 s+4$ if and only if

$$
\begin{aligned}
& (s+2)(s+1) a\left(\tau_{s},\left[\bullet, \tau_{s+1}\right]\right)=(s+1) a\left(\tau_{s}, \tau_{s+3}\right)+(s+2) a\left(\tau_{s},\left[\tau_{s+2}\right]\right), \\
& (s+2)(s+1) a\left(\tau_{s+1},\left[\tau_{s+1}\right]\right)=(s+2) a\left(\tau_{s+1}, \tau_{s+2}\right)+s(s+2) a\left(\tau_{s},\left[\tau_{s+2}\right]\right)-s a\left(\tau_{s}, \tau_{s+3}\right) .
\end{aligned}
$$

Proof. The simplifying assumptions imply that for conjugate symplecticity up to order $2 s+2$ only

$$
a\left(\tau_{s}, \tau_{s+1}\right)=-s c\left(\tau_{s}, \tau_{s}\right)
$$

has to be satisfied. This is always possible with a suitable choice of $c\left(\tau_{s}, \tau_{s}\right)$. 
The conditions for conjugate symplecticity up to order $2 s+3$ are

$$
\begin{gathered}
a\left(\tau_{s}, \tau_{s+3}\right)=-(s+2) c\left(\tau_{s}, \tau_{s+2}\right)-\left(\begin{array}{c}
s+2 \\
3
\end{array}\right) c\left(\tau_{s}, \tau_{s}\right), \\
a\left(\tau_{s},\left[\tau_{s+2}\right]\right)=-(s+1) c\left(\tau_{s},\left[\tau_{s+1}\right]\right)-\left(\begin{array}{c}
s+1 \\
3
\end{array}\right) c\left(\tau_{s},\left[\tau_{s-1}\right]\right), \\
a\left(\tau_{s},\left[\bullet, \tau_{s+1}\right]\right)=-s c\left(\tau_{s},\left[\bullet, \tau_{s}\right]\right)-c\left(\tau_{s},\left[\tau_{s+1}\right]\right)-\left(\begin{array}{l}
s \\
3
\end{array}\right) c\left(\tau_{s},\left[\bullet, \tau_{s-2}\right]\right)-\left(\begin{array}{l}
s \\
2
\end{array}\right) c\left(\tau_{s},\left[\tau_{s-1}\right]\right), \\
a\left(\tau_{s+1}, \tau_{s+2}\right)=-s c\left(\tau_{s}, \tau_{s+2}\right)-(s+1) c\left(\tau_{s+1}, \tau_{s+1}\right)-s\left(\begin{array}{c}
s+1 \\
2
\end{array}\right) c\left(\tau_{s}, \tau_{s}\right), \\
a\left(\tau_{s+1},\left[\tau_{s+1}\right]\right)=-s c\left(\tau_{s},\left[\tau_{s+1}\right]\right)-s c\left(\tau_{s+1},\left[\tau_{s}\right]\right)-s\left(\begin{array}{l}
s \\
2
\end{array}\right) c\left(\tau_{s},\left[\tau_{s-1}\right]\right) .
\end{gathered}
$$

By the simplifying assumption $C(s)$ we have $c\left(\tau_{s},\left[\tau_{s-1}\right]\right)=\frac{1}{s-1} c\left(\tau_{s}, \tau_{s}\right), c\left(\tau_{s},\left[\bullet, \tau_{s}\right]\right)=$ $\frac{1}{s} c\left(\tau_{s}, \tau_{s+2}\right), c\left(\tau_{s},\left[\bullet, \tau_{s-2}\right]\right)=\frac{1}{s-2} c\left(\tau_{s}, \tau_{s}\right)$ and $c\left(\tau_{s+1},\left[\tau_{s}\right]\right)=\frac{1}{s} c\left(\tau_{s+1}, \tau_{s+1}\right)$. Elimination of the four free parameters for the coefficients $c$ from the six relations yields two conditions for conjugate symplecticity up to order $2 s+4$. This completes the proof of the theorem.

\section{Applications}

We apply the above criteria for conjugate symplecticity to important classes of integration methods. Recall that for $s$-stage Runge-Kutta methods the simplifying assumptions can be written as

$$
\begin{aligned}
& C(\eta): \sum_{j=1}^{s} a_{i j} c_{j}^{k-1}=\frac{c_{i}^{k}}{k}, \quad k=1, \ldots, \eta, \quad i=1, \ldots, s, \\
& D(\zeta): \sum_{i=1}^{s} b_{i} c_{i}^{k-1} a_{i j}=\frac{b_{j}}{k}\left(1-c_{j}^{k}\right), \quad k=1, \ldots, \zeta, j=1, \ldots, s .
\end{aligned}
$$

For the computation of the expressions $a(u, v)$, appearing in Section 5 , it is convenient to write the Runge-Kutta coefficients in terms of orthogonal polynomials. This is closely related to the W-transformation of Hairer \& Wanner (1996, Section IV.5).

\subsection{Lobatto IIIA methods}

Lobatto IIIA methods are Runge-Kutta collocation methods whose nodes $c_{1}=0, c_{2}, \ldots, c_{s-1}, c_{s}=1$ are the zeros of the polynomial $P_{S}(t)-P_{s-2}(t)$, where $P_{S}(t)$ denotes the shifted Legendre polynomial of degree $s$ (Lobatto quadrature, see Appendix C). They are symmetric methods of order $2 s-2$, and they satisfy the simplifying assumptions $C(s)$ and $D(s-2)$. The special case $s=2$ is the implicit trapezoidal rule, which is conjugate to the symplectic implicit midpoint rule. We consider here the case $s \geqslant 3$. 
The Runge-Kutta coefficients $a_{i j}$ can be expressed in terms of Legendre polynomials as follows:

$$
a_{i j}=\sum_{l=1}^{s-1}(2 l-1) \int_{0}^{c_{i}} P_{l-1}(x) \mathrm{d} x b_{j} P_{l-1}\left(c_{j}\right),
$$

where $b_{j}$ are the weights of the Lobatto quadrature. To prove this formula we have to check the condition $C(s)$, which uniquely determines the coefficients $a_{i j}$. Details are given in Appendix C. There it is also shown how the B-series coefficients of the Lobatto IIIA method of order $2 s-2$ can be obtained. They satisfy

$$
a\left(\tau_{s-1}, \tau_{s+2}\right)=\frac{\left(s^{2}-2\right) s !(s-1) !((s-2) !)^{2}}{4(2 s-3)((2 s-2) !)^{2}}, \quad a\left(\tau_{s-1},\left[\tau_{s+1}\right]\right)=\frac{s !((s-1) !)^{2}(s-2) !}{4(2 s-3)((2 s-2) !)^{2}} .
$$

Combining these formulas with Theorem 5.9 gives the following result.

THEOREM 6.1 For $s \geqslant 3$ the Lobatto IIIA method of order $2 s-2$ is conjugate symplectic up to order $2 s$, but it is not conjugate symplectic up to a higher order.

\subsection{Lobatto IIIB methods}

Lobatto IIIB methods are also based on Lobatto quadrature. They are symmetric, of order $2 s-2$, and they satisfy the simplifying assumptions $C(s-2)$ and $D(s)$. For $s=2$ the method is equivalent to the symplectic implicit midpoint rule. We consider here the case $s \geqslant 3$.

Expressing the Runge-Kutta coefficients in terms of Legendre polynomials yields

$$
a_{i j}=\sum_{l=1}^{s-1}(2 l-1) P_{l-1}\left(c_{i}\right) b_{j} \int_{c_{j}}^{1} P_{l-1}(x) \mathrm{d} x .
$$

These coefficients are uniquely defined by $D(s)$, which is verified in Appendix C. The B-series coefficients of the Lobatto IIIB Runge-Kutta method of order $2 s-2$ satisfy, for $s \geqslant 3$,

$$
a\left(\tau_{s-1}, \tau_{s}\right)=-\left(\frac{(s-1) !(s-2) !}{(2 s-2) !}\right)^{2}, \quad a\left(\tau_{s-1},\left[\tau_{s-1}\right]\right)=0 .
$$

Combining these formulas with Theorem 5.10 gives the following result.

THEOREM 6.2 For $s \geqslant 3$ the Lobatto IIIB method of order $2 s-2$ is not conjugate symplectic up to an order higher than $2 s-2$.

\subsection{Energy-preserving collocation methods}

The energy-preserving variant of collocation methods was introduced in Hairer (2011). It can be interpreted as an implicit Runge-Kutta method with a continuum of stages. For a differential equation $\dot{y}=f(y)$ it can be written as

$$
Y_{\tau}=y_{0}+h \int_{0}^{1} A_{\tau, \sigma} f\left(Y_{\sigma}\right) \mathrm{d} \sigma, \quad y_{1}=y_{0}+h \int_{0}^{1} B_{\tau} f\left(Y_{\tau}\right) \mathrm{d} \tau .
$$


We have $B_{\sigma}=A_{1, \sigma}$ so that $y_{1}=Y_{1}$, and the coefficients $A_{\tau, \sigma}$ are polynomials of degree $s-1$ in $\sigma$, defined by the simplifying assumption $\int_{0}^{1} A_{\tau, \sigma} \sigma^{k-1} \mathrm{~d} \sigma=\frac{\tau^{k}}{k}, k=1, \ldots, s$, which is equivalent to $C(s)$. An explicit formula for these coefficients (see Brugnano et al., 2010) is given with the help of the shifted Legendre polynomials as follows:

$$
A_{\tau, \sigma}=\sum_{l=1}^{s}(2 l-1) \int_{0}^{\tau} P_{l-1}(\alpha) \mathrm{d} \alpha \cdot P_{l-1}(\sigma), \quad B_{\tau}=1, \quad C_{\tau}=\int_{0}^{1} A_{\tau, \sigma} \mathrm{d} \sigma=\tau .
$$

The method is a B-series integrator $y_{1}=B\left(a, y_{0}\right)$ that is of order $2 s$, symmetric and satisfies the simplifying assumptions $C(s)$ and $D(s-1)$. We know from Hairer (2011) that the method is conjugate symplectic up to order $2 s+2$. Can it be conjugate symplectic up to a higher order? For this purpose we compute the B-series coefficients (see Appendix B). For the method of order $2 s$ we have, for $s \geqslant 1$,

$$
\begin{gathered}
a\left(\tau_{s}, \tau_{s+3}\right)=\frac{(s+2)^{2}(s+1)(s !)^{4}}{4(2 s+3)(2 s+1) s((2 s) !)^{2}}, \\
a\left(\tau_{s},\left[\tau_{s+2}\right]\right)=\frac{(s+1)^{2}(s !)^{4}}{4(2 s+1)^{2} s((2 s) !)^{2}}, \\
a\left(\tau_{s},\left[\bullet, \tau_{s+1}\right]\right)=\frac{\left(s^{2}+2 s-1\right)(s !)^{4}}{4(2 s-1)(2 s+1) s((2 s) !)^{2}} .
\end{gathered}
$$

These formulas show that the first condition of Theorem 5.11 is violated. We thus have the following result (note that the statement for $s=1$ was proved in Celledoni et al., 2009).

THEOREM 6.3 The energy-preserving collocation method of order $2 s$ is conjugate symplectic up to order $2 s+2$, but it is not conjugate symplectic up to a higher order.

\section{Summary of results}

In Table 2 we summarize the results of the present article, and we put them into the context of further classes of implicit Runge-Kutta methods. The last two columns indicate whether the method is symplectic and what is the order of conjugate symplecticity. The 'Gauss' methods are collocation methods of maximal order $2 s$, and they are known to be symplectic (Hairer et al., 2006, Section VI.4).

TABLE 2 Overview on the order of conjugate symplecticity

\begin{tabular}{lclcc}
\hline Method & Order & Simplifying assumptions & Symplecticity & Conjugate symplecticity \\
\hline Gauss & $2 s$ & $C(s), D(s)$ & Yes & $\infty$ \\
Radau IA & $2 s-1$ & $C(s-1), D(s)$ & No & $2 s-1$ \\
Radau IIA & $2 s-1$ & $C(s), D(s-1)$ & No & $2 s-1$ \\
Trapezoidal rule & 2 & $C(2)$ & No & $\infty$ \\
Lobatto IIIA, $s \geqslant 3$ & $2 s-2$ & $C(s), D(s-2)$ & No & $2 s$ \\
Implicit midpoint rule & 2 & $C(1), D(1)$ & Yes & $\infty$ \\
Lobatto IIIB, $s \geqslant 3$ & $2 s-2$ & $C(s-2), D(s)$ & No & $2 s-2$ \\
Lobatto IIIC, $s \geqslant 2$ & $2 s-2$ & $C(s-1), D(s-1)$ & No & $2 s-2$ \\
$\begin{array}{l}\text { Energy-preserving } \\
\quad \text { collocation }\end{array}$ & $2 s$ & $C(s), D(s-1)$ & No & $2 s+2$ \\
\hline
\end{tabular}


For the harmonic oscillator $\dot{y}=\mathrm{i} y$, the numerical solution of a Runge-Kutta method is given by $y_{n+1}=R(\mathrm{i} h) y_{n}$, where $R(z)$ is its stability function. This linear mapping is symplectic if and only if $R(z) R(-z)=1$. It is conjugate symplectic up to order $r$ if and only if $R(z) R(-z)=1+\mathcal{O}\left(z^{r+1}\right)$. For the simple differential equation $\dot{y}=\mathrm{i} y$ the composition of B-series integrators commutes, so that the stability functions of $\Phi_{h}$ and $\chi \circ \Phi_{h} \circ \chi^{-1}$ are identical. The fact that the stability function of the methods 'Radau IA', 'Radau IIA' and 'Lobatto IIIC' are subdiagonal Padé approximations proves that these methods are neither symplectic nor conjugate symplectic up to an order higher than that of the stability function.

It is interesting to mention that the underlying one-step method of linear multistep methods cannot be symplectic, but it is conjugate symplectic up to an arbitrarily high order (cf. Hairer, 2008) if the method is symmetric.

A natural question is the investigation of energy-preserving integrators that are conjugate symplectic (up to arbitrarily high order). Their existence as formal B-series is known and follows from the fact that symplectic integrators conserve a modified Hamiltonian and modified quadratic first integrals (see Chartier et al., 2006). It is still a challenge to find a computational method (i.e., an integrator that can be implemented) that exactly preserves the energy and is conjugate symplectic at the same time. The methods of Section 6.3 do not share these properties.

\section{Acknowledgements}

We would like to thank Ander Murua for making available his Mathematica package for the manipulation of rooted trees and B-series. It is very helpful for checking the formulas obtained for $s$ that are not too large. We further thank him and Gilles Vilmart for commenting on an early version of the manuscript.

\section{Funding}

Fonds National Suisse (Project No. 200020-126638).

\section{REFERENCES}

Brugnano, L., Iavernaro, F. \& Trigiante, D. (2010) Hamiltonian boundary value methods (energy conserving discrete line integral methods). J. Numer. Anal. Ind. Appl. Math., 5, 17-37.

Butcher, J. C. (1972) An algebraic theory of integration methods. Math. Comput., 26, 79-106.

Butcher, J. C. \& CHAN, T. M. H. (2002) A new approach to the algebraic structures for integration methods. BIT, 42, 477-489.

Celledoni, E., Mclachlan, R. I., Mclaren, D. I., Owren, B., Quispel, G. R. W. \& Wright, W. M. (2009) Energy-preserving Runge-Kutta methods. Math. Model. Numer. Anal., 43, 645-649.

Celledoni, E., Mclachlan, R. I., Owren, B. \& QuisPel, G. R. W. (2010) Energy-preserving integrators and the structure of B-series. Found. Comput. Math., 10, 673-693.

Chartier, P., Faou, E. \& Murua, A. (2006) An algebraic approach to invariant preserving integrators: The case of quadratic and Hamiltonian invariants. Numer. Math., 103, 575-590.

Chartier, P., Hairer, E. \& Vilmart, G. (2010) Algebraic structures of B-series. Found. Comput. Math., 10, 407-427.

Ge, Z. \& MARSDEN, J. E. (1988) Lie-Poisson Hamilton-Jacobi theory and Lie-Poisson integrators. Phys. Lett. $A, \mathbf{1 3 3}, 134-139$.

HAIRER, E. (2008) Conjugate-symplecticity of linear multistep methods. J. Comput. Math., 26, 657-659.

HAIRER, E. (2011) Energy-preserving variant of collocation methods. J. Numer. Anal. Ind. Appl. Math., 5, 73-84. 
Hairer, E., Lubich, C. \& WAnner, G. (2006) Geometric Numerical Integration. Structure-Preserving Algorithms for Ordinary Differential Equations. Springer Series in Computational Mathematics, vol. 31, 2nd edn. Berlin: Springer.

HAIRER, E. \& WANner, G. (1974) On the Butcher group and general multi-value methods. Computing, 13, 1-15. HaIrer, E. \& WAnner, G. (1996) Solving Ordinary Differential Equations II. Stiff and Differential-Algebraic Problems. Springer Series in Computational Mathematics, vol. 14, 2nd edn. Berlin: Springer.

LEONE, P. (2000) Symplecticity and symmetry of general integration methods. Ph.D. Thesis, Université de Genève, Section de Mathématiques.

Murua, A. (1999) Formal series and numerical integrators. I. Systems of ODEs and symplectic integrators. Appl. Numer. Math., 29, 221-251.

\section{Appendix A. Shifted Legendre polynomials}

We consider Legendre polynomials shifted to the interval $[0,1]$. By abuse of notation, we continue to write $P_{k}(\tau)$ for the polynomial of degree $k$. The polynomials are normalized by $P_{k}(1)=1$, they satisfy the orthogonality relations

$$
\int_{0}^{1} P_{k}(x) P_{j}(x) \mathrm{d} x=\left\{\begin{array}{cc}
0 & \text { if } k \neq j \\
(2 j+1)^{-1} & \text { if } k=j
\end{array}\right.
$$

and they can conveniently be computed from Rodrigues' formula,

$$
P_{k}(x)=\frac{(-1)^{k}}{k !} \frac{\mathrm{d}^{k}}{\mathrm{~d} x^{k}}\left(x^{k}(1-x)^{k}\right) .
$$

The integral of the Legendre polynomial satisfies, for $k \geqslant 1$,

$$
\int_{0}^{x} P_{k}(t) \mathrm{d} t=\frac{1}{2(2 k+1)}\left(P_{k+1}(x)-P_{k-1}(x)\right),
$$

and repeated integration by parts gives the relation

$$
\int_{0}^{1} P_{j}(x) x^{k} \mathrm{~d} x=k(k-1) \cdots(k-j+1) \frac{k !}{(k+j+1) !} .
$$

To express the product $x P_{k}(x)$ as a linear combination of Legendre polynomials, we use the three-term recurrence relation

$$
(k+1) P_{k+1}(x)=(2 k+1)(2 x-1) P_{k}(x)-k P_{k-1}(x) .
$$

\section{Appendix B. Technical details for the energy-preserving collocation method}

We explain the verification of formula (6.6). The other formulas can be checked with the same techniques. The B-series coefficients of a Runge-Kutta method (6.5) with a continuum of stages are obtained as for classical Runge-Kutta methods with sums replaced by integrals. In this way we get

$$
a\left(\tau_{s+3} \circ \tau_{s}\right)=\int_{0}^{1} \int_{0}^{1} B_{\tau} C_{\tau}^{s+2} A_{\tau, \sigma} C_{\sigma}^{s-1} \mathrm{~d} \sigma \mathrm{d} \tau=\frac{1}{s(2 s+3)},
$$


where we have exploited the simplifying assumption $C(s)$. Using explicit formulas for the coefficients $A_{\tau, \sigma}, B_{\tau}$ and $C_{\tau}$, we obtain

$$
\begin{aligned}
a\left(\tau_{s} \circ \tau_{s+3}\right) & =\int_{0}^{1} \int_{0}^{1} B_{\tau} C_{\tau}^{s-1} A_{\tau, \sigma} C_{\sigma}^{s+2} \mathrm{~d} \sigma \mathrm{d} \tau \\
& =\int_{0}^{1} \tau^{s-1} \sum_{l=1}^{s}(2 l-1) \int_{0}^{\tau} P_{l-1}(\alpha) \mathrm{d} \alpha \int_{0}^{1} P_{l-1}(\sigma) \sigma^{s+2} \mathrm{~d} \sigma \mathrm{d} \tau .
\end{aligned}
$$

With the expansion of $\alpha^{s+2}$ into a series of Legendre polynomials,

$$
\alpha^{s+2}=\sum_{l=1}^{s+3}(2 l-1) P_{l-1}(\alpha) \int_{0}^{1} P_{l-1}(\sigma) \sigma^{s+2} \mathrm{~d} \sigma,
$$

we obtain

$$
\begin{aligned}
a\left(\tau_{s} \circ \tau_{s+3}\right) & =\int_{0}^{1} \tau^{s-1} \int_{0}^{\tau}\left(\alpha^{s+2}-\sum_{l=s+1}^{s+3}(2 l-1) P_{l-1}(\alpha) \int_{0}^{1} P_{l-1}(\sigma) \sigma^{s+2} \mathrm{~d} \sigma\right) \mathrm{d} \alpha \mathrm{d} \tau \\
& =\frac{1}{(s+3)(2 s+3)}-R(s)
\end{aligned}
$$

where

$$
R(s)=\sum_{l=s+1}^{s+3}(2 l-1)\left(\int_{0}^{1} \tau^{s-1} \int_{0}^{\tau} P_{l-1}(\alpha) \mathrm{d} \alpha \mathrm{d} \tau\right)\left(\int_{0}^{1} P_{l-1}(\sigma) \sigma^{s+2} \mathrm{~d} \sigma\right) .
$$

From (A.3) and the orthogonality relation, the expression in the first parentheses vanishes for $l>s+1$. Only the term with $l=s+1$ remains and yields

$$
R(s)=-\frac{((s-1) !(s+2) !)^{2}}{4(2 s-1) !(2 s+3) !}
$$

Since the quadrature conditions $a\left(\tau_{k}\right)=1 / k, k \geqslant 1$, are satisfied for all energy-preserving B-series integrators, we obtain $a\left(\tau_{s}, \tau_{s+3}\right)=a\left(\tau_{s} \circ \tau_{s+3}\right)+a\left(\tau_{s+3} \circ \tau_{s}\right)-a\left(\tau_{s}\right) a\left(\tau_{s+3}\right)=-R(s)$. This proves formula (6.6). The other expressions are obtained in a similar way.

\section{Appendix C. Lobatto methods}

The nodes of the Lobatto quadrature are the zeros of the polynomial $P_{S}(x)-P_{s-2}(x)$, and they satisfy $c_{1}=0$ and $c_{s}=1$. The quadrature formula is of order $2 s-2$, which means that polynomials of degree $\leqslant 2 s-3$ are integrated without error. The dominant error term is given by

$$
\sum_{i=1}^{s} b_{i} c_{i}^{2 s-2}-\frac{1}{2 s-1}=\frac{s !(s-1) !(s-1) !(s-2) !}{(2 s-1) !(2 s-2) !}
$$


Further useful formulas are

$$
\begin{aligned}
\sum_{i=1}^{s} b_{i} P_{l-1}\left(c_{i}\right) P_{k-1}\left(c_{i}\right) & =0 \quad \text { if } l+k \text { is odd }, \\
\sum_{i=1}^{s} b_{i} P_{s-1}\left(c_{i}\right) P_{s-1}\left(c_{i}\right) & =\frac{1}{s-1}, \\
\sum_{i=1}^{s} b_{i} P_{s}\left(c_{i}\right) P_{s}\left(c_{i}\right) & =\frac{1}{2 s-3}, \\
\sum_{i=1}^{s} b_{i} P_{s-1}\left(c_{i}\right) P_{s+1}\left(c_{i}\right) & =\frac{2 s-1}{(2 s-3)(s+1)(s-1)},
\end{aligned}
$$

which can be obtained by using properties of the Legendre polynomials (see Appendix A) and by using the fact that the nodes of the Lobatto quadrature satisfy $P_{s}\left(c_{i}\right)=P_{s-2}\left(c_{i}\right)$ for all $i$.

\section{C.1 Lobatto IIIA methods}

For the verification of formula (6.1) we compute

$$
\sum_{j=1}^{s} a_{i j} P_{k-1}\left(c_{j}\right)=\sum_{l=1}^{s-1}(2 l-1) \int_{0}^{c_{i}} P_{l-1}(x) \mathrm{d} x \sum_{j=1}^{s} b_{j} P_{l-1}\left(c_{j}\right) P_{k-1}\left(c_{j}\right)=\int_{0}^{c_{i}} P_{k-1}(x) \mathrm{d} x .
$$

The second equality holds for $k \leqslant s$ because the quadrature formula is exact for polynomials of degree $\leqslant 2 s-3$ and because we have $\int_{0}^{c_{i}} P_{s-1}(x) \mathrm{d} x=0$ as a consequence of (A.3). This proves the identity $\sum_{j=1}^{s} a_{i j} p\left(c_{j}\right)=\int_{0}^{c_{i}} p(x) \mathrm{d} x$ for all polynomials of degree $s-1$. Putting $p(x)=x^{k-1}$ verifies the condition $C(s)$ that uniquely determines the coefficients $a_{i j}$ of the method.

For the computation of the B-series coefficients (6.2) we use the relation

$$
\sum_{i=1}^{s} b_{i} c_{i}^{s-2} a_{i j}-\frac{b_{j}}{s-1}\left(1-c_{j}^{s-1}\right)=\frac{(s-1) !(s-2) !}{(2 s-2) !} b_{j} P_{s-1}\left(c_{j}\right),
$$

which permits a simplification similar to that with the condition $D(s-1)$. This formula can either be proved with the help of (6.1) or by writing the left-hand expression as a linear combination of $P_{0}\left(c_{i}\right), P_{1}\left(c_{i}\right), \ldots, P_{s-1}\left(c_{i}\right)$ and computing the coefficients with help of the orthogonality relations. A direct calculation then gives

$$
\begin{gathered}
a\left(\tau_{s-1}, \tau_{s+2}\right)=\frac{(s-1) !(s-2) !}{(2 s-2) !} \sum_{j=1}^{s} b_{j} P_{s-1}\left(c_{j}\right) c_{j}^{s+1}, \\
a\left(\tau_{s-1},\left[\tau_{s+1}\right]\right)=\frac{(s-1) !(s-2) !}{(2 s-2) !} \sum_{j=1}^{s} b_{j} P_{s-1}\left(c_{j}\right) \sum_{k=1}^{s} a_{j k} c_{k}^{s} .
\end{gathered}
$$

Writing $c_{j}^{s+1}$ (respectively $\sum_{k=1}^{s} a_{j k} c_{k}^{s}$ ) as a linear combination of $P_{0}\left(c_{j}\right), \ldots, P_{s-1}\left(c_{j}\right)$ finally yields the relations (6.2). Note that only the coefficient of $P_{s-1}\left(c_{j}\right)$ is relevant. 


\section{C.2 Lobatto IIIB methods}

To prove formula (6.3) we compute, for $k=1, \ldots, s$,

$$
\sum_{i=1}^{s} b_{i} P_{k-1}\left(c_{i}\right) a_{i j}=\sum_{l=1}^{s-1}(2 l-1) \sum_{i=1}^{s} b_{i} P_{k-1}\left(c_{i}\right) P_{l-1}\left(c_{i}\right) b_{j} \int_{c_{j}}^{1} P_{l-1}(x) \mathrm{d} x=b_{j} \int_{c_{j}}^{1} P_{k-1}(x) \mathrm{d} x .
$$

The same argument as before proves that the coefficients (6.3) verify condition $D(s)$.

Most of the B-series coefficients for the Lobatto IIIB methods, needed in Section 6.2, can be reduced via the simplifying assumption $D(s)$ to the bushy trees $\tau_{k}, k \leqslant 2 s-1$. For the remaining tree $\left[\tau_{s-1}, \tau_{s-1}\right]$ we use the relation

$$
\sum_{j=1}^{s} a_{i j} c_{j}^{s-2}-\frac{c_{i}^{s-1}}{s-1}=-\frac{(s-1) !(s-2) !}{(2 s-2) !} P_{s-1}\left(c_{i}\right)
$$

which has a similar effect to the simplifying assumption $C(s-1)$. It can be proved in the same way as its analogue for the Lobatto IIIA methods. This relation enables formulas (6.4) to be obtained. 